\title{
The Research on Status Management of Environmental Testing Equipment Based on PHM Technology
}

\author{
Bin Liu ${ }^{1}$, a , Ming $X \mathrm{u}^{1}$ and Youshuang $\mathrm{Yu}{ }^{1}$ \\ ${ }^{1}$ Baicheng Ordnance Test Center, Baicheng, Jilin,China, 137001 \\ aliubinshore@163.com
}

\begin{abstract}
Keywords: Prognostics and Health Management (PHM); environmental testing equipment; status management.
\end{abstract}

Abstract. Based on the connotation and function Prognostics and Health Management (PHM) technology, the feasibility of PHM applied in status management of environmental testing equipment was analyzed. The status management system of environmental testing equipment based on PHM was proposed, in which the functions and prediction methods in the system were introduced.

\section{Introduction}

In the environment simulation test, environmental testing equipment is the indispensable tool for artificial simulation testing. Since 1970, as the rapid development of environmental testing equipment, it gradually becomes a comprehensive, complicate interdisciplinary subject. Because the performance of environmental testing equipment is improved, complexity and integration is enhanced, as well as the system construction for environmental simulation testing is promoted, the traditional methods for preventive maintenance guarantee cannot fit for the current and complicated environment simulation testing.

In America, during the Joint Strike Fighter (Joint Strike Fighter, JSF) was designed, the advanced technology of Prognostics and Health Management (PHM) [1] is introduced, which can significantly reduce the cost of maintenance, use and support, and improve equipment safety and availability. PHM applied in status management of environmental testing equipment, will greatly improve the management level of environmental testing equipment, and enhance support capabilities of environmental simulation testing.

\section{The Content and function of PHM}

In PHM, status data is collected with advanced data acquisition sensors, and features are extracted with a variety of algorithms to acquire health status features in system, which can achieve the monitoring, forecasting and management of system health status. PHM represents a method of transformation for fault prediction and status management, which means that traditional diagnosis based on sensors transform to prediction based on intelligent system, and reactive communication transforms to pilot type 3R (ie, taking the right managment to the right part at the right time).

The key of PHM is that using as few sensors (such as eddy current sensor, low-power wireless integrated micro-sensors, wireless micro-electro-mechanical systems, etc.) as possible to collect data.With the various algorithms (such as Labor transform, fast Fourier transform, discrete Fourier Transform) and intellgent models (such as expert systems, neural networks, fuzzy logic, etc.), the system health status is evaluated. Before the system failure is carried out, the failure can be predicted. Combining with a variety of available resources information, a range of maintenance guarantee measures are provided for the system maintenance depended on the condition.

The main functions of PHM include: fault detection, fault isolation, fault prediction, remaining service life prediction, parts life tracking, performance degradation trend tracking, warranty tracking, failure selective reporting (according to the type of fault, classification information reports to management personnel ), decision support and resource management, fault tolerance, information integration and inference engine and information management (the right information informs right 
person at the right time).PHM is not intended to eliminate the fault, but to understand and predict when the fault occurs and achieve the pre-fault repairs.

\section{Open process of PHM}

As PHM technology applied in the aerospace, military and industrial fields, different types of PHM system are emerged, and the basic idea is similar. Open system architecture for condition-based maintenance (OSA-CBM) combines common design ideas and the techniques and methods in PHM systems [2], which formed open process of PHM composed by seven-part flow (Figure 1).

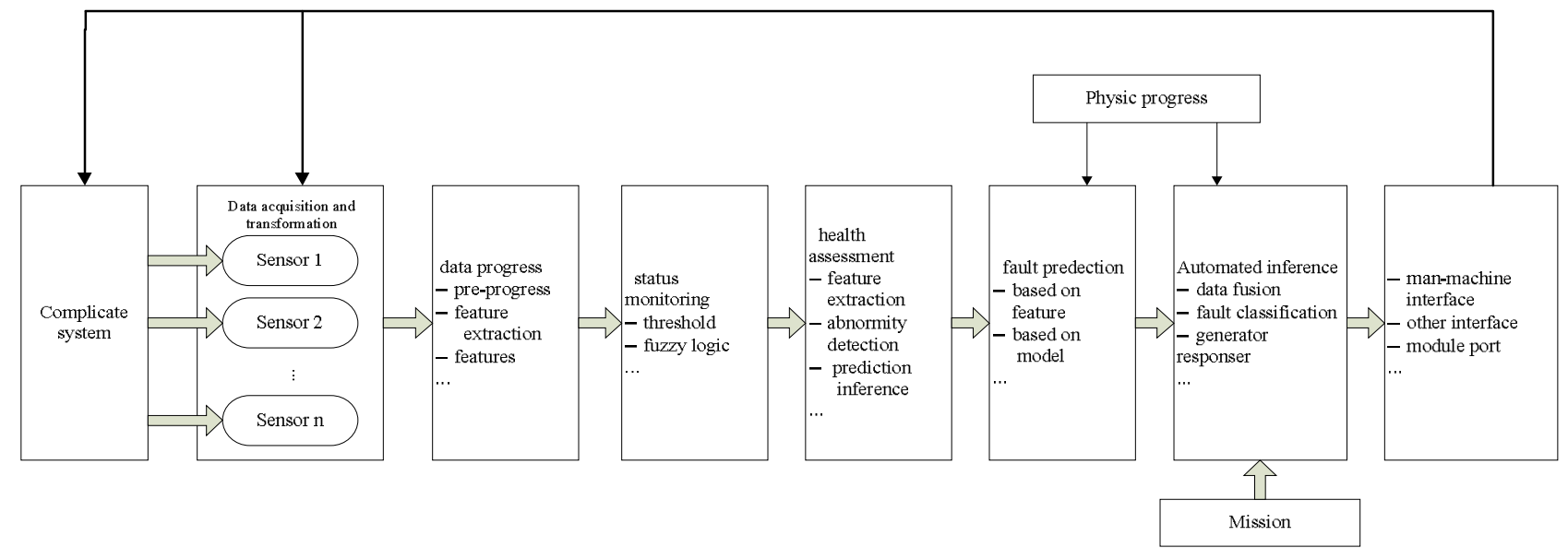

Figure 1 open process of PHM based on OSA-CBM

The processing operation of each module in PHM flow can be seen in Figure 1. It should be noted that, there is no obvious boundary between each module, and cross-feedback of data information is existed. For example, the threshold value of condition monitoring can be adjust based on the results of fault diagnosis and predition module, or the classifier parameters can be adjust based on the performance of diagnosis and prediction.

\section{Status management system of environmental testing equipment based on PHM}

System Structure. Status management system of environmental testing equipment based on PHM is divided into three parts and seven levels: the first part is constructed by the various sensors distributed in the testing device subsystem. The second part is composed of data processing layer and subsystems detection layer. The third part is constructed by the layers of health assessment, fault detection, decision support, output system. Figure 2 showed the relationship.

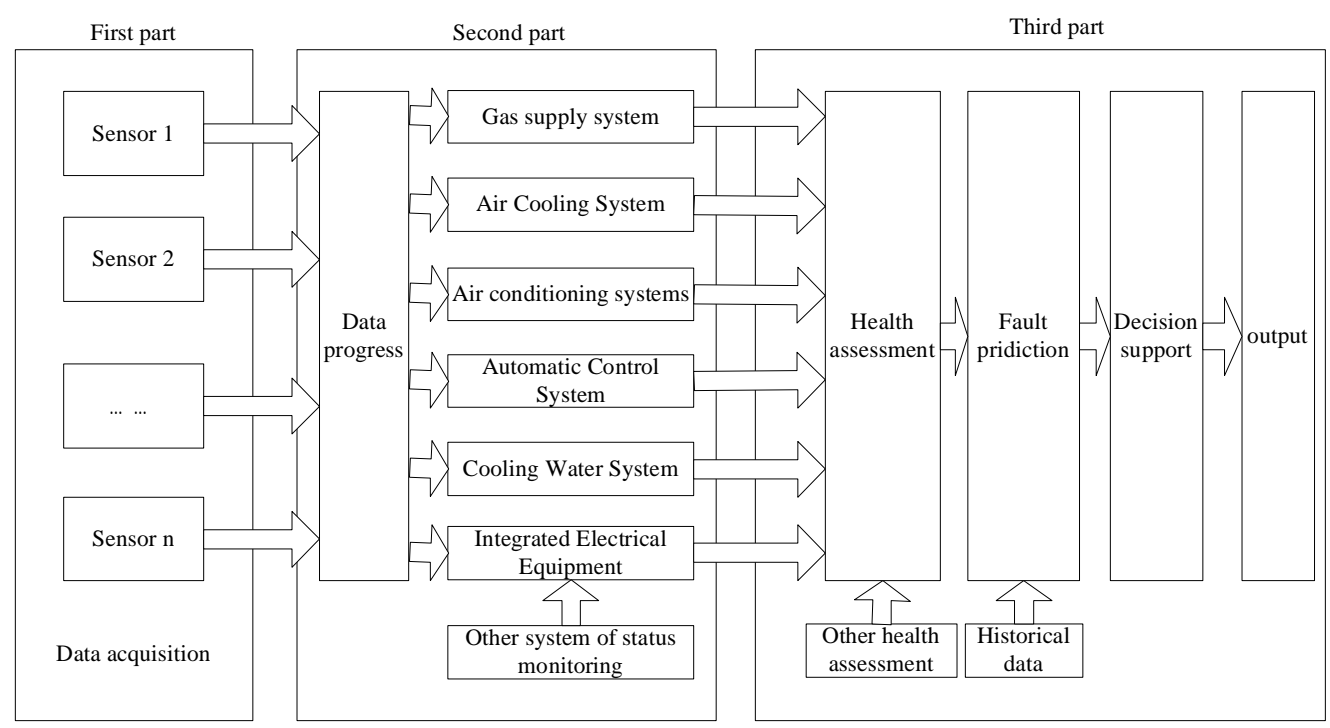

Figure 2 the system structure 
The layers of data acquisition, data processing, status monitoring and health assessment are located on system platform of environmental test equipment. The layers of failure prediction, decision support and output system are achieved mainly wth the corresponding health management subsystem / equipment.

System Function. On the layer of data acquisition, sensors are connected with specific physical measuring equipment of environmental testing equipment to collect signals from the data bus, for providing the raw data.

The layer of data processing can process the raw data, and with some feature extraction algorithm, the raw data can be converted into the acquired forms for condition monitoring, health assessment and failure prediction.

The layer of condition monitoring receives data from the sensors, data processing layer and other condition monitoring modules, and the system state-related characteristics are calculated and estimated. Compared with a predetermined failure criterion, the data is used for monitoring the current system state, and according to predetermined values of extremums / thresholds, fault alarm is provided. Each state detection module has the function of signal processing, information integration and regional reasoning, which is a real-time actuator for the continuous monitoring of operating conditions in corresponding subsystem.

The layer of health assessment receives data from different state monitoring module and other health assessment module. Based on the output of state monitoring layer and historical assessed value, the health status of monitoring system, subsystem or component are assessed, and determined whether these systems should be downgraded. If the health state of the system is downgraded, the layer will produce diagnostic information, and indicate that fault may occur.

The layer of failure prediction can integrate data from above layers, and assess the future health of the monitoring systems, subsystems and components. If the system detectes any part or subsystem performance degradation, it will isolate the fault, evaluate fail time, and give the plan about completion for the necessary managment work.

The layer of decision support receives the data from the layer state monitoring, health assessment and forecast, and make the appropriate decision support based on the output of the previous layers, which can provide support for maintenance resources management and other health management.

The output layer have the ability to communicate with all the other layers, and received transmitted information from each functional module, send data to automated management information systems of test equipment which is able to judge the safety of test equipment, arrange test tasks, manage technique status, update status records of test equipment, adjust use plan, generate the project of maintenance work, and analyze the technique state of entire testing equipment.

Achievement of health assessment and prediction method. Working state of environmental testing equipment has the fetures of "long period of storage, boot time in a row". The reliability can be predicted accurately, and accurate and reliable state information is provided for environmental simulation testing. The state management system of environmental testing equipment based on PHM uses intelligent inference engine (such as expert systems, neural networks, machine learning, fuzzy logic and genetic algorithms) for reasoning the reliability and health.

In recent years, support vector machine (SVM) based on statistical theory has good generalization capability and global optimal solution, which can develop rapidly. Based on limited samples, it has balance between the model complexity and learning ability to get the best generalization ability for solving the problem of small sample learning [3], and has been widely used in fault classification, state prediction and other fields. In order to improve the operation speed, reduce redundant information, the proposed system has combined rough set (RS) theory with SVM. RS theory can effectively analyze and deal with imprecise, inconsistent, incomplete information, find hidden knowledge, and reveal the potential law. Combined with SVM, RS front-end system was designed to eliminate redundant status. Use the information states preprocessed with RS method as training samples, and prediction inference system of SVM state is shown in Figure 3. 


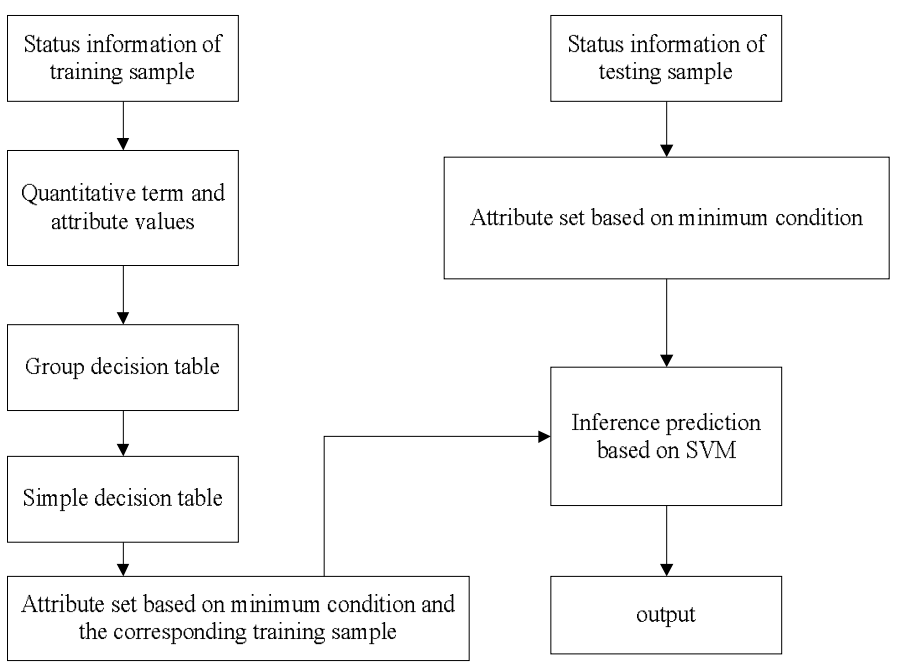

Figure 3 prediction inference system of SVM states.

State management system of environmental testing equipment based on PHM constantly collects signal for analysis and information fusion. Then decisions and suggestions are output, and send to information processing system, which can judge the reliability of components, implement the management of device state, update status records, adjust use plans, and evaluate the health status of component or system.

In the prediction inference process, the process of determination from component failure to system failure is the most uncertain step. Because of great variable and strong nonlinear, the results of intelligent inference engine often requires algorithms of track and trend to determine the health status to quantify the health and damage levels of output environment test equipment.

\section{Conclusion}

It is a novel method that state management status management based on PHM technology is applied for quality detected of environmental testing equipment. PHM technology can accurately manage the safety operation and maintenance of environmental simulation testing equipment. Ensuring equipment reliability and maintainability, with reducing spare parts, detected frequency, and staffing, the security costs can be reduced. The risk of failure is reduced, and the rate of task completion is improved, which is important for maintenance guarantee of environment simulation testing equipment.

\section{References}

[1] Ning Dongfang, Zhang Weiguo, Li Bin. Technology of Prognostics and Health Management. Aeronautical manufacturing Technology, 2009(5): 71-73.

[2] Discenzo F M , Nickerson W , Mitchell CF , et al. in: Open systems architecture enables health management for next generation system monitoring and maintenance. USA: Rockwell Autumation,2001.

[3] Tong Zhige, Guo Chuan, Li Yinghui. Application Research on the PHM Technology in the Advanced Fight Plane Maintain and Guarantee. Aviation Maintenance \& engineering, 2009(1): 37-39. 